\title{
Reducing morbidity from uterovaginal prolapse in Nepalese women through surgical camps: an ambitious approach.
}

\author{
Ashma Rana, \\ Dept Ob/Gyn, TU Teaching Hospital, \\ Kathmandu, Nepal.
}

Uterovaginal prolapse is something more than merely falling down of womb, as it has been occasionally found in association with vaginal or cervical carcinoma (fig1) in women living with this problem for many years. ${ }^{1}$ Even today there are cases of vesicovaginal fistula coexisting together with prolapsed uterus as a poor consequence of obstructed labor. Rectovaginal fistula has also been formed from the forgotten ring pessary giving an insight in to cumulated cases of uterovaginal prolapse. ${ }^{2}$

In consideration of diverse geographical topography and the nature of the clinical or community based study carried out in general population against the women who are sick and actual sufferers during different period of year, prevalence of POP (pelvic organ prolapse) is quoted to be as high as $25.1 \%$ [A clinic based study done in far western Nepal, (FHD, GTZ AND UNFPA 2000)] Similar research findings catering the sick women in a western territory 'Bhajang' discovered $18 \%$ of the total 530 to be affected. ${ }^{3}$

Whereas it is surprising not to find any cases of uterine prolapse in Manang, a mountainous areas and ditto in Jumla another mountainous region with nil documentation of prolapse from the Institute of Medicine led community based reproductive morbidity (unpublished) study [(funded by UNFPA /WHO) May $28-31,2006]$ probably due to rice plantation time, in the latter. $^{4}$

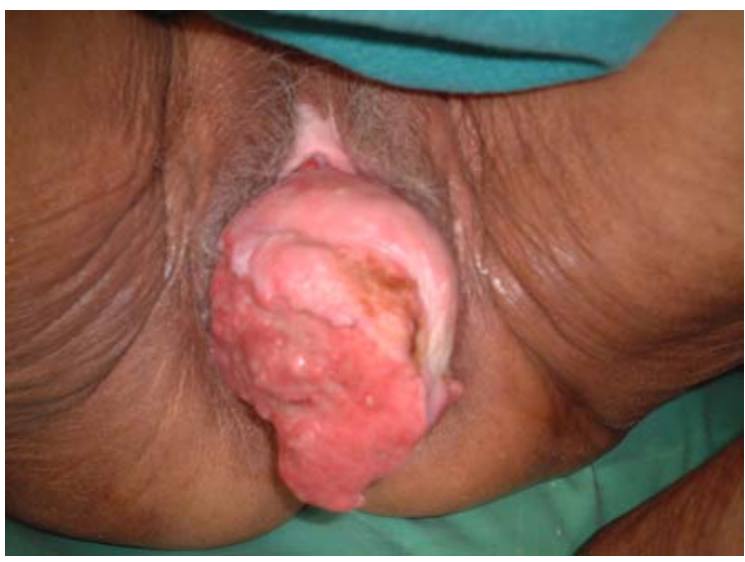

Fig. 1 Carcinoma cervix in a prolapsed uterus in a 91 year old lady from a gynaecological camp.
If the "no prolapse" scenario is the outcome of surgical camps conducted throughout the country in the past few years, governmental and various non governmental organizations should be acknowledged for their effective role in providing the financial support to the surgical team who are the main heroes coping to work in most difficult situation within limited resources.

As of the status today, uterovaginal surgical camps are mushrooming every year with extension of services in various parts throughout the country. This is hoped to reduce the prevalence of prolapse and the related magnitude of morbidity which had been addressed in one of the annual meeting of our national society. ${ }^{5}$

The concept of surgical camps is marvelous but the approaches are undoubtedly less palatable. Honestly, the sad part is when the women have to be flown or driven in cases of dire emergency arising from lately detected postoperative complication like significant hemoperitoneum or ill appreciated vaginal vault hematoma.

Surgeons tackling such complicated cases at the referral centers with wider choices in better equipped anesthetic and ICU facility view with optimism that such complication could have been avoided with thrust given on quality of work not on the quantities. Usually each surgical camp is assigned a target of 100 cases within a short frame work of time; say a week including travel time. By and large 16-22 surgeries are daily performed in an average by 2-3 doctors working at a stretch in the hectic schedule starting at $6-8$ am and going up to $6-8 \mathrm{pm}$, incorporating staff nurses as first assistants (for simple cases) and running 2-3 parallel operation tables simultaneously by a single anesthetist. The situation can be improved by installing an improvised surgical team consisting of more than 2 -3 doctors who neither have to race down nor instigate competition to the next operating surgeons operating in the adjacent operation tables, laid across the same room. The country would soon see the decrease in number of cumulated cases of uterovaginal prolapse with annual coverage of at least 500 cases in turn building surgical expertise of the budding surgeons as MD Ob/Gyn Residents have also been able to acquire 
tremendous surgical exposure having had the opportunity of performing 15 vaginal hysterectomies during the surgical camp.

These kinds of surgical camps for major vaginal surgery at community and district level must be the first of its kind and needs applause. If there is a global consensus that every woman should enjoy reproductive rights, why should our women sufferers who have walked up and down for several days in search of remedies, be provided a services even beyond the national standards, when most of the funding agencies are international? It is imperative that the operating surgeons screen the cases themselves, besides being only occupied for surgery, such that cases of pregnancy don't go unrecognized and uterus lodging a fetus does not get removed during pregnancy. At the same time, difficult and time consuming surgery (huge procidentia or dermoid/myoma complicating prolapse) can be passed for a lighter day with lesser case loads (fig 2). Poorly selected cases have come dead, the moral of the story being "rather women live with prolapse than die from the surgery".

Surgeons are at least expected to take post operative rounds so that the complications are timely detected, this might eventually help to reduce the related morbidity from hemoperitoneum before DIC sets in, when frantically fresh frozen plasma, platelet rich plasma have to be looked for. While dealing with such kind of emergencies everyone wishes that the case was well prepared preoperatively. Our experience of loaded

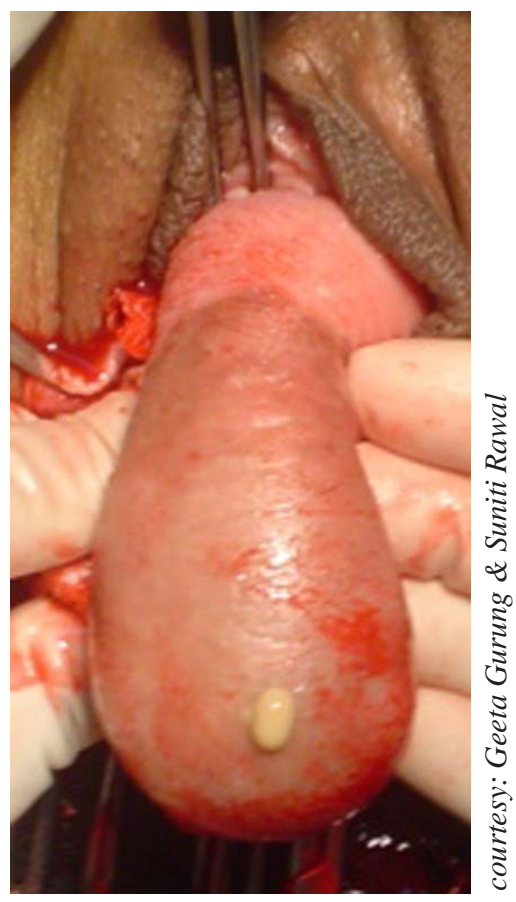

Fig.2. Vaginal dermoid complicating a case of Uterovaginal prolapse at a surgical camp: bowel (sigmoid) during exploration of cuff haematoma at relaparotomy was bad which could have been minimized by instituting at least a soap water enema which is generally not advocated in camp surgery when most of the Terai dwellers are habitual constipaters.

Finally restriction of cases per surgeon per day is mandatory so that the surgery is undertaken at a comfortable pace. The government has restricted the number of mini laparotomy per surgeon per day and this should be also applied in vaginal prolapse surgery although the two surgery is not comparable.

With the major concerns that any bladder injury does not go undetected (hear say evidences of postoperative genito-urinary fistula having undergone repair) and any bleeders do not go unrecognized (that is inclined to provoke worrisome coagulopathy), it is necessary that the operating team must be led by a gynecologist, a full fledged doctor as the first assistant and residents / interns who could be employed as the second assistant.

By this subtle approach general fears of transforming a lesser type of reproductive morbidity from uterovaginal prolapse to more moribund forms like peritonitis/ pyoperitoneum (there are many alleged cases) and more distressing ones like fistula can be avoided. One must rethink, how empathetic are we? Many women with associated rectal prolapse are being served by Thiersch's operation with time as pretext.

A country with major geographical disadvantages, Nepal, yet has been looming with innumerable measures in improving women's health needs targeted at women who cannot make to the big cities by the extension of services in remote areas of the community by the organization of surgical health camps and reaching to masses of women who have in due course of time adjusted to live with prolapse. This is an ambitious approach, the services imparting the reproductive right to women where the women would benefit more if they did not have to be air lifted in face of dire emergencies meeting the provision of general anesthesia facility. Or there would be more than just one anesthetist to support three OT tables who would be able to cope up with every situation simultaneously faced by skilled gynecologists (now no more dearth in the country). Moreover post operative case follow up must be maintained, enlisting all the complications, so measures can be adopted to avoid them.

Responsibility does not impede here just by complying the surgical remedies when there will be another woman in every corner of the country bearing down on her own, pushing out the baby unattended. With such scenario of self assisted delivery in cowshed, kitchen (warmer place) and under staircase rampantly met, one would speculate whether the number of prolapse cases could come to a decline. Besides this, there is persistent 
puerperal under care that has remained stagnant, where women are expected to carry water and loads of firewood under the background of inadequate nutrition. How can these problems be eradicated? [As verified from the Thesis study 2004-2005: Dept of Ob/ Gyn, IOM which has identified $81 \%$ of women to have been affected with uterine prolapse of reproductive origin, i.e. initiating from child birth].Or are we inviting the birth of yet another prolapse? Based on our own observation at TU Teaching Hospital of only a decimal of women affected by prolapse following hospital delivery and almost negligible after caesarean (having come across only a single case after three caesarean) acquaints us with the fact that the crux in the genesis of uterovaginal prolapse to be the unattended deliveries in Nepalese women, only 7-9\% women having an access to supervised deliveries. ${ }^{6}$

To wrap up, a supervised delivery not depriving women access to basic health care or economic empowerment that does not deny adequate puerperal rest and accessibility to enriched diet supportive for tissue collagen going par with the experiences gained from improvised surgical expertise in dealing with women living in real distress due to suffering from uterovaginal prolapse by the help of well organized prolapse surgical camps aiming more than targeted surgery, related reproductive morbidity from prolapse uterus in Nepalese women is envisioned to be reduced by far in the decades to come.

\section{References}

1. Gurung P, Rana A, Pradhan N, Shah R, Giri K, Manandhar T. Vaginal carcinoma in total prolapse Nepal Medical College Journal 2001; 3 (1): 46-7

2. Giri K, Khakurel MP, Rana A. Rectovaginal fistula due to prolong use of ring pessary: a case report. JSSN 2000; 3(2): 58-59

3. Tuladhar H. An overview of reproductive health of women in Bajhang district: Nepal Med Coll J 2005; 7(2):107-11

4. Pant PR, Singh KP, Sharma NR, Giri P, Shrestha $K$, Kuwar K. None with uterovaginal prolapse in Manag Gynecological Camp. Gynaecology and Obstetric annual health letter 2003. 9: 59- 60.

5. Rana A. Utero-vaginal prolapses as a gynecological morbidity in TUTH. Abstract in Souvenir: 6th National Conference NESOG, 21st March, 2000. Katmandu, Nepal. p25

6. Rana A, Gurung G, Pradhan N. Prolapse and problem. JSSN 2001; 4 (1).32-37 